\title{
An algorithm for the reduction of linear DAE
}

\author{
M.P. Quéré \\ LITP-IBP \\ Univ. Paris 6 \\ 4 Pl. Jussieu F-75252 Paris Cedex 05 \\ et LMC-IMAG \\ quere@posso.ibp.fr
}

\author{
G. Villard \\ LMC-IMAG \\ 46 av. Félix Viallet \\ F-38031 Grenoble Cedex \\ Gilles.Villard@imag.fr
}

\section{Abstract}

We study linear Differential Algebraic Equations, DAE, with time varying coefficients. Such equations

$$
B(t) \dot{x}(t)=A(t) x(t)+f(t)
$$

are intensively studied from a numerical point of view. Canonical forms have been proposed to find conditions under which the equation admits a solution, to find the set of consistent initial conditions and to determine conditions under which there is a unique solution. However, since the situation where the system admits infinitely many solutions for one initial value is not really tractable in a numerician framework, few algorithms may be found in this latter case. Among them, we find the method of P. Kunkel and $V$. Mehrmann who propose a new set of local characterizing quantities for the treatment of the system. This leads to a generalization of the global index.

Nevertheless, these latter characterizing quantities impose too restrictive conditions on the input equations. We propose new definitions for them that lead to a new algorithm which puts the initial system into a reduced form without doing any assumption on it. This allows us to propose a new generalization of the global index and a definition for the singularities of the initial system. The questions of existence and uniqueness of solutions are solved in all interval which does not contain singularity.

Finally, since from a practical point of view the general case of analytic functions is difficult to handle, we focus on the polynomial case. We propose an effective algorithm that has been implemented and report some experiments.

\section{Introduction}

Given a system:

$$
A(t) \dot{x}(t)+B(t) x(t)=f(t)
$$

where $A(t)$ and $B(t)$ are $n \times p$ matrices with analytic components on an interval $I$ and $f(t)$ is undefined, we want to know under which conditions the system (1) has solutions,

Permission to copy without fee all or part of this material is granted provided that the copiss ars not made or distributed for direct commercial advantages, the ACM copyright notice and the title of the publication and its date appear, and notice is given that copying is by permission of the Association for Computing Machinery. To copy otherwise, or to republish, requires a fee and/or specific permission. ISSAC'95 - 7/95 Montreal, Canada

(C) 1995 ACM 0-89791-699-9/95/0007 $\$ 3.50$ and whether these are uniquely determined by their value at any $t_{0} \in I$. Finally, we want to find the set of consistent initial conditions, i.e. conditions $x\left(t_{0}\right)=x_{0}, t_{0} \in I$ for which the corresponding initial value problem has at least one solution.

When $A(t)$ is a regular square matrix on $I$, system (1) turns out to be an ODE:

$$
\dot{x}(t)+A^{-1}(t) B(t) x(t)=A^{-1}(t) f(t)
$$

In this case, we can answer the previous questions.

Otherwise, (1) is called a Differential Algebraic Equation or DAE. To introduce the key notion of index, let us assume for instance that $A(t)$ is a square matrix of the form

$$
\left\|\begin{array}{cc}
A_{1}(t) & 0 \\
0 & 0
\end{array}\right\|
$$

where $A_{1}(t)$ is a square regular matrix on $I$. System (1) can be written as:

$$
\begin{aligned}
& A_{1}(t) \dot{x}_{1}(t)+B_{11}(t) x_{1}(t)+B_{12}(t) x_{2}(t)=f_{1}(t) \\
& B_{21}(t) x_{1}(t)+B_{22}(t) x_{2}(t)=f_{2}(t)
\end{aligned}
$$

that is a set of algebraic and differential equations. Assume now that $f_{2}(t)$ can be differentiated. Differentiating the second row of (2) we obtain the system:

$$
\begin{aligned}
& \left\|\begin{array}{cc}
A_{1}(t) & 0 \\
B_{21}(t) & B_{22}(t)
\end{array}\right\|\left\|\begin{array}{l}
\dot{x}_{1}(t) \\
\dot{x}_{2}(t)
\end{array}\right\|+ \\
& \left\|\begin{array}{cc}
B_{11}(t) & B_{12}(t) \\
\dot{B}_{21}(t) & \dot{B}_{22}(t)
\end{array}\right\|\left\|\begin{array}{l}
x_{1}(t) \\
x_{2}(t)
\end{array}\right\|=\left\|\begin{array}{l}
f_{1}(t) \\
\dot{f}_{2}(t)
\end{array}\right\| .
\end{aligned}
$$

If the matrix $B_{22}(t)$ is regular, this new system is an ODE, else we have another DAE and the process can be iterated. The number of differentiations of the initial DAE that are required to generate an $O D E$, when possible, is called the differential index of the DAE. Gear [10] gives a general definition of the differential index. We write it here in the linear case.

Definition 1 The differential index $i_{d}$ of a system (1) is defined by:

- if $A(t)$ is regular on $I, i_{d}=0$.

- Otherwise consider the system of equations

$$
\begin{aligned}
& A(t) \dot{x}(t)+B(t) x(t)=f(t) \\
& \frac{d}{d t}(A(t) \dot{x}(t)+B(t) x(t))=A(t) x^{(2)}(t)+ \\
& \quad(\dot{A}(t)+B(t)) \dot{x}(t)+\dot{B}(t) x(t)=\dot{f}(t) \\
& \cdots \\
& \frac{d^{s}}{d t^{s}}(A(t) \dot{x}(t)+B(t) x(t))=\frac{d^{s} f(t)}{d t^{s}}
\end{aligned}
$$


as a linear system of equations in the separate dependent variables $\dot{x}, x^{(2)}, \ldots, x^{(s+1)}$, and solve for these variables as functions of $x$ and $t$ considered as independent variables. If it is possible to solve for $\dot{x}$ for some finite $s$, then, the index $i_{d}$ is defined as the smallest $s$ for which (3) can be solved for $\dot{x}(x, t)$.

This differential index is a fundamental notion from a numerical point of view. In particular when $i_{d} \geq 2$, the numerical computation of solutions requires specific care [8]. As we will see, the latter definition can be extended to the cases where it does not exist a $s$ such that the system is solvable.

From now we distinguish a regular and a singular case. System (1) is said to be regular (or analytically solvable following the definition in [3]) on the interval $I$ if for any sufficiently smooth $f(t)$, there exist solutions to (1) defined on all $I$ which are uniquely determined by their value at any $t_{0} \in I$.

For this kind of DAE, Campbell and Petzold [3] have shown that there exists a pair of nonsingular analytic matrices on $I, P(t)$ and $Q(t)$, such that the transformation $x(t)=Q(t) y(t)$ and left multiplication by $P(t)$ turn (1) into

$$
\begin{aligned}
& \left\|\begin{array}{cc}
I d & 0 \\
0 & N(t)
\end{array}\right\|\left\|\begin{array}{l}
\dot{y}_{1}(t) \\
\dot{y}_{2}(t)
\end{array}\right\|+ \\
& \left\|\begin{array}{cc}
C(t) & 0 \\
0 & I d
\end{array}\right\|\left\|\begin{array}{c}
y_{1}(t) \\
y_{2}(t)
\end{array}\right\|=\left\|\begin{array}{l}
f_{1}(t) \\
f_{2}(t)
\end{array}\right\|
\end{aligned}
$$

where $N(t)$ is nilpotent and lower (or upper) triangular.

Form (4) is called standard canonical form (or SCF). When a system is in SCF, the solutions and the consistent initial conditions can be found. For a regular DAE, Campbell and Petzold also give an algorithm to compute $P(t)$ and $Q(t)$. Besides, we can find algorithms $[8,2]$ which compute the differential index of a system and establish its regularity. Thus, the regular case is well known.

The singular case is more complicated. Indeed, a singular system may have more than one solution for a consistent initial condition; also, there is not always a solution for any $f(t)$ smooth as it may be. Kunkel and Mehrmann [13, 14] have proposed an algorithm for singular systems. They have extended the definition of differential index (strangeness index) when (3) cannot be solved for $\dot{x}$ for any finite $s$. Unfortunately, there are some DAE's, some even regular, that cannot be solved with this approach.

Our aim in this paper is to generalize the approach of Kunkel and Mehrmann to enlarge the classification of the systems in case of analytic coefficients. This will lead us to generalize also the index and to a natural definition for the singularities of (1). We want to point out that our goal is not to propose a general theory for DAE. We just propose an efficient algorithm which allows to simplify linear DAE. It is obvious that theoretical works has been done on the subject, both from a geometrical point of vew (see $[16,22]$ ) and in differential algebra (see $[4,5]$ ). These aproaches are general but often lack efficiency.

In section 1 we begin with classical definitions of equivalence and basic linear algebra results. The algorithm of Kunkel and Mehrmann is briefly described in section 2. We explain why it is too restrictive according to us. In section 3 , we present a new algorithm that brings the system into a reduced form. Under this form, the system will be called full system. The point is that the form can be computed for every system (1) on the whole of its definition interval. In section 4 , we show how the form computed allows us to decide of the existence and uniqueness of the solutions. In most cases, this can be decided except for a set of isolated points. To describe this phenomenon, we introduce the notion of singularity of a DAE. In this section we also extend the definition of the differential index. Finally, in section 5, we study the polynomial case and show that the algorithm can be easily implemented. Some experiments are reported using MAPLE.

Notation We often use the notation $(A(t), B(t))$ for the system (1).

\section{Basic definitions and theorems}

In this section we define algebraic and differential equivalence of two DAE and then give two theorems for matrix transformations.

\subsection{Equivalence between DAE}

Given a DAE $A(t) \dot{x}(t)+B(t) x(t)=f(t)$ defined on the interval $I$, transformations of the form

$$
E q_{i} \longleftarrow E q_{i}+a(t) E q_{j}
$$

where $E q_{i}$ and $E q_{j}$ are respectively the $i t h$ and $j t h$ equations of the system and $a(t)$ is analytic on $I$, does not change the solutions of the system. This kind of transformation amounts to a left multiplication by a matrix $P(t)$ analytic and nonsingular on $I$.

If we set now $x(t)=Q(t) y(t)$ where $Q(t)$ is analytic and nonsingular on $I$, we do not change the nature (ie the dimension of the solution manifold and the existence of singularity) of the solutions either. The system obtained can be written:

$$
A(t) Q(t) \dot{y}(t)+(A(t) \dot{Q}(t)+B(t) Q(t)) y(t)=f(t)
$$

These two transformations are purely algebraic, we say that the two systems

$$
\begin{gathered}
A(t) \dot{x}(t)+B(t) x(t)=f(t) \text { and } \\
\tilde{A}(t) \dot{x}(t)+\tilde{B}(t) x(t)=\tilde{f}(t)
\end{gathered}
$$

are algebraically equivalent on $I$ if there exist two matrices $P(t)$ and $Q(t)$ analytic and nonsingular on $I$ such that:

$$
\begin{aligned}
& P(t) A(t) Q(t)=\tilde{A}(t) \\
& P(t) B(t) Q(t)+P(t) A(t) \dot{Q}(t)=\bar{B}(t)
\end{aligned}
$$

We will also need differential operations like:

$$
\begin{aligned}
& E q_{i} \longleftarrow E q_{i}+a(t) \dot{E} q_{j} \text { and } \\
& E q_{n+1} \longleftarrow E q_{j}
\end{aligned}
$$

where $n$ is the number of equations, $E q_{i}, E q_{\jmath}$ and $a(t)$ are defined as above.

We say that two systems are differentially equivalent on $I$ if we can obtain the second one by applying (6) or (7) to the first one. In this definition, the operations (6) and (7) must let the order of the system unchanged.

Definition 2 Two systems of DAE $S$ and $\tilde{S}$ are equivalent on $I$ if there exists systems $S_{0}=S, S_{1}, \ldots, S_{k}=\tilde{S}$ such that for all $i, 0 \leq i \leq k-1, S_{i}$ and $S_{i+1}$ are algebraically equivalent or differentially equivalent on $I$. 


\subsection{Two very useful theorems}

Preliminary remark: An analytic function defined on $I$ which is equal to zero on an interval $J \subset I$ is equal to zero on the whole of $I$. Consequently, if $M(t)$ is an $n \times p$ matrix with analytic entries on $I$ and has rank $r(t)$, then $r(t)$ is a constant on $I$ except at some isolated points.

The following two theorems form essential steps both in Kunkel and Mehrmann's algorithm and in our algorithm. The proofs are given in [21].

Theorem 1 If $M(t)$ is real analytic on $I$ and $r \geq \operatorname{Rank}(M(t))$ for all $t \in I$, then there exists real analytic nonsingular matrices $P(t)$ and $Q(t)$ such that:

$P(t) M(t) Q(t)=\left\|\begin{array}{cc}\Sigma(t) & 0 \\ 0 & 0\end{array}\right\|$ with $\Sigma(t)$ a $r \times r$ matrix

Theorem 2 If $\Sigma(t)$ is a $r \times r$ real analytic matrix on $I$ and $\operatorname{Rank}(\Sigma(t))=r(t)$ is equal to $r$ except at some isolated $t \in I$, then there exists a real analytic nonsingular matrix $P(t)$ such that:

$$
P(t) \Sigma(t)=\Delta(t)
$$

where $\Delta(t)$ is upper triangular.

This last factorization is called, in the numerical linear algebra community, a rank revealing QR factorization.

\section{The Kunkel and Mehrmann algorithm}

In this section we briefly describe the Kunkel and Mehrmann algorithm for the reduction of linear DAE. Further details may be found in $[13,14]$.

To give their algorithm, Kunkel and Mehrmann define three characteristic quantities $r, a$ and $s$. Let $(A(t), B(t))$ be a linear DAE with analytic components on $I$. Assume that $\operatorname{Rank}(A(t))=r$ is constant on $I$, by theorem 1 , the system is equivalent to the following one:

$$
\left(\left\|\begin{array}{cc}
I d_{r} & 0 \\
0 & 0
\end{array}\right\|,\left\|\begin{array}{cc}
B_{11}(t) & B_{12}(t) \\
B_{21}(t) & B_{22}(t)
\end{array}\right\|\right)
$$

where $I d_{r}$ is the identity block of order $r$.

Assume now that $\operatorname{Rank}\left(B_{22}(t)\right)=a$ is constant on $I$, applying theorem 1 for $M(t)=B_{22}(t)$ we obtain:

$$
\left(\left\|\begin{array}{ccc}
I d_{r} & 0 & 0 \\
0 & 0 & 0 \\
0 & 0 & 0
\end{array}\right\|,\left\|\begin{array}{ccc}
B_{11}(t) & B_{12}(t) & B_{13}(t) \\
B_{21}(t) & I d_{a} & 0 \\
B_{31}(t) & 0 & 0
\end{array}\right\|\right)
$$

where the $B_{i j}(t)$ are different from the previous ones.

Finally, assume that $\operatorname{Rank}\left(B_{31}(t)\right)=s$ is constant on $I$, we put the system into the form:

$$
\begin{aligned}
& \left(\left\|\begin{array}{cccc}
I d_{s} & 0 & 0 & 0 \\
0 & I d_{d} & 0 & 0 \\
0 & 0 & 0 & 0 \\
0 & 0 & 0 & 0 \\
0 & 0 & 0 & 0
\end{array}\right\|\right. \\
& \left.\left\|\begin{array}{cccc}
B_{11}(t) & B_{12}(t) & B_{13}(t) & B_{14}(t) \\
B_{21}(t) & B_{22}(t) & B_{23}(t) & B_{24}(t) \\
B_{31}(t) & B_{32}(t) & I d_{a} & 0 \\
I d_{s} & 0 & 0 & 0 \\
0 & 0 & 0 & 0
\end{array}\right\|\right)
\end{aligned}
$$

with $d=r-s$
Using algebraic transformations we can replace the blocks $B_{13}(t), B_{23}(t), B_{31}(t), B_{32}(t), B_{11}(t), B_{21}(t)$ and $B_{22}(t)$ by zero (see [13]). If $r, a$ and $s$ are constant on $I$ the initial system is equivalent to a system of the form:

$$
\left(\left\|\begin{array}{cccc}
I d_{s} & 0 & 0 & 0 \\
0 & I d_{d} & 0 & 0 \\
0 & 0 & 0 & 0 \\
0 & 0 & 0 & 0 \\
0 & 0 & 0 & 0
\end{array}\right\|,\left\|\begin{array}{cccc}
0 & B_{12}(t) & 0 & B_{14}(t) \\
0 & 0 & 0 & B_{24}(t) \\
0 & 0 & I d_{a} & 0 \\
I d_{s} & 0 & 0 & 0 \\
0 & 0 & 0 & 0
\end{array}\right\|\right)
$$

We also can write this system:

$$
\begin{cases}(a) & \dot{x}_{1}(t)+B_{12}(t) x_{2}(t)+B_{14} x_{4}(t)=g_{1}(t) \\ (b) & \dot{x}_{2}(t)+B_{24}(t) x_{4}(t)=g_{2}(t) \\ (c) & x_{3}(t)=g_{3}(t) \\ (d) & x_{1}(t)=g_{4}(t) \\ (e) & 0=g_{5}(t)\end{cases}
$$

where there exists a nonsingular analytic matrix $P(t)$ such that $g(t)=P(t) f(t)$.

We now differentiate equation (8d) and insert it in (8a), which then becomes an algebraic equation. The new system is equivalent to the initial one in the sense of definition 2, and can be written:

$$
\left(\left\|\begin{array}{cccc}
0 & 0 & 0 & 0 \\
0 & I d_{d} & 0 & 0 \\
0 & 0 & 0 & 0 \\
0 & 0 & 0 & 0 \\
0 & 0 & 0 & 0
\end{array}\right\|,\left\|\begin{array}{cccc}
0 & B_{12}(t) & 0 & B_{14}(t) \\
0 & 0 & 0 & B_{24}(t) \\
0 & 0 & I d_{a} & 0 \\
I d_{s} & 0 & 0 & 0 \\
0 & 0 & 0 & 0
\end{array}\right\|\right)
$$

We can apply again the same transformations, computing new characteristic values $r, a$ and $s$. Repeating this procedure, this gives rise to a sequence of systems $\left(A_{i}(t), B_{i}(t)\right)$ and to the related sequences of characteristic values $\left(r_{i}, a_{i}, s_{i}\right)$. However these transformations are possible if $r_{i}, a_{i}$ and $s_{i}$ are constant on $I$ at each step. In that case, Kunkel and Mehrmann [13] proved there exists a number $m$, called the strangeness index, defined by:

$$
m=\min \left\{i \in \mathbf{N} \mid s_{i}=0\right\} .
$$

When $m$ is reached the iterations are stopped. In addition, they proved that the sequences of characteristic values have the properties:

$$
\begin{array}{llll}
r_{i}>r_{i+1} & \text { for } i<m, & r_{i}=r_{m} & \text { for } i \geq m \\
a_{i}<a_{i+1} & \text { for } i<m, & a_{i}=a_{m} & \text { for } i \geq m \\
s_{i} \geq s_{i+1} & \text { for } i<m, & s_{i}=0 & \text { for } i \geq m
\end{array}
$$

Then the initial system $A(t) \dot{x}(t)+B(t) x(t)=f(t)$ is equivalent to a system under the form:

$$
\left(\left\|\begin{array}{ccc}
I d_{r_{m}} & 0 & 0 \\
0 & 0 & 0 \\
0 & 0 & 0
\end{array}\right\|,\left\|\begin{array}{ccc}
0 & 0 & B_{13}(t) \\
0 & I d_{a_{m}} & 0 \\
0 & 0 & 0
\end{array}\right\|\right)
$$

where inhomogeneity is determined by $f(t), \dot{f}(t), \ldots, f^{(m)}(t)$. According to Kunkel and Mehrmann there exists a relation between the strangeness index and the differentiation index $i_{d}$ when they are both defined:

$$
i_{d}= \begin{cases}0 & \text { for } m=0, a_{0}=0 \\ m+1 & \text { otherwise }\end{cases}
$$

In addition, when the system is in the form (10), it is very easy to answer to the existence and uniqueness of the solutions and the consistency of initial conditions. However, this 
algorithm can be improved. A lot of systems (even regular ones) do not fulfill the condition $r_{i}, a_{i}$, and $s_{i}$ constant for all $i, 0 \leq i \leq m$ on the definition interval $I$. We present two examples...

Example 1 If $f(t) \in C^{2}(\mathbf{R})$, the system

$\left\|\begin{array}{ll}0 & t \\ 0 & 0\end{array}\right\|\left\|\begin{array}{c}\dot{x}_{1}(t) \\ \dot{x}_{2}(t)\end{array}\right\|+\left\|\begin{array}{cc}1 & 0 \\ 0 & 1\end{array}\right\|\left\|\begin{array}{l}x_{1}(t) \\ x_{2}(t)\end{array}\right\|=\left\|\begin{array}{c}f_{1}(t) \\ f_{2}(t)\end{array}\right\|$

has a unique solution on all $\mathbf{R}$ which is

$$
\begin{aligned}
& x_{1}(t)=f_{1}(t)-t \dot{f}_{2}(t) \\
& x_{2}(t)=f_{2}(t) .
\end{aligned}
$$

However, the Kunkel and Mehrmann algorithm cannot say anything at $t=0$ because $r_{0}=1$ on all $\mathbf{R}$ except at $t=0$.

Example 2 If $f(t) \in C^{2}(\mathbf{R})$ and satisfies the consistency condition $t\left(f_{1}(t)-\dot{f}_{3}(t)\right)=f_{2}(t)$ the system

$$
\left\|\begin{array}{ll}
1 & 0 \\
0 & 0 \\
0 & 0
\end{array}\right\|\left\|\begin{array}{c}
\dot{x}_{1}(t) \\
\dot{x}_{2}(t)
\end{array}\right\|+\left\|\begin{array}{cc}
0 & 1 \\
0 & t \\
1 & 0
\end{array}\right\|\left\|\begin{array}{l}
x_{1}(t) \\
x_{2}(t)
\end{array}\right\|=\|\| \begin{gathered}
f_{1}(t) \\
f_{2}(t) \\
f_{3}(t)
\end{gathered} \|
$$

has a unique solution on all $\mathbf{R}$ which is

$$
\begin{aligned}
& x_{1}(t)=f_{3}(t) \\
& x_{2}(t)=f_{1}(t)-\dot{f}_{3}(t) .
\end{aligned}
$$

However, the Kunkel and Mehrmann's algorithm cannot say anything at $t=0$ because $a_{0}=1$ on all $\mathbf{R}$ except at $t=0$.

We propose in the next section a generalization of the Kunkel and Mehrmann's algorithm which allows us to treat systems on all their definition interval.

\section{Our generalized algorithm}

A careful study of the Kunkel and Mehrmann's algorithm shows that the assumptions about $r_{i}$ and $a_{i}$ for all $i, 0 \leq$ $i \leq m$, are superfluous. If we just assume that $r_{m}$ and $a_{m}$ (which is the case for the last two examples) are constant on $I$, we can apply the Kunkel and Mehrmann's algorithm in the same way. As for to the assumptions about the $s_{i}$, even if they are not constant, we can differentiate the corresponding equations and add them to the system. The new algorithm is based on these remarks.

\section{Generalized algorithm:}

Let the initial system $A(t) \dot{x}(t)+B(t) x(t)=f(t)$ be defined on $I$. Like in the Kunkel and Mehrmann algorithm, with three applications of theorem 1 , we can put the system into the equivalent form:

$$
\begin{aligned}
& \left(\left\|\begin{array}{cccc}
A_{11}(t) & A_{12}(t) & 0 & 0 \\
A_{21}(t) & A_{22}(t) & 0 & 0 \\
0 & 0 & 0 & 0 \\
0 & 0 & 0 & 0 \\
0 & 0 & 0 & 0
\end{array}\right\|,\right. \\
& \left.\left\|\begin{array}{cccc}
B_{11}(t) & B_{12}(t) & B_{13}(t) & B_{14}(t) \\
B_{21}(t) & B_{22}(t) & B_{23}(t) & B_{24}(t) \\
B_{31}(t) & B_{32}(t) & \Gamma_{a}(t) & 0 \\
0 & \Omega_{s}(t) & 0 & 0 \\
0 & 0 & 0 & 0
\end{array}\right\|\right)
\end{aligned}
$$

where $A_{i j}(t)$ (respectively $\Gamma_{a}(t)$ and $\Omega_{s}(t)$ ) is an $r \times r$ (respectively $a \times a$ and $s \times s)$ square matrix, nonsingular on $I$ except at some isolated points. Applying theorem 2 we can put the $A_{i j}$ block into an upper triangular form ${ }^{1}$ :

$$
\begin{gathered}
\left(\left\|\begin{array}{cccc}
\Sigma_{11}(t) & \Sigma_{12}(t) & 0 & 0 \\
0 & \Sigma_{22}(t) & 0 & 0 \\
0 & 0 & 0 & 0 \\
0 & 0 & 0 & 0 \\
0 & 0 & 0 & 0
\end{array}\right\|,\right. \\
\left.\left\|\begin{array}{cccc}
B_{11}(t) & B_{12}(t) & B_{13}(t) & B_{14}(t) \\
B_{21}(t) & B_{22}(t) & B_{23}(t) & B_{24}(t) \\
B_{31}(t) & B_{32}(t) & \Gamma_{a}(t) & 0 \\
0 & \Omega_{s}(t) & 0 & 0 \\
0 & 0 & 0 & 0
\end{array}\right\|\right)
\end{gathered}
$$

Now we can add to the system the derivative of the equation $\Omega_{s}(t) x_{2}(t)=g_{4}(t)$ to get the equivalent system

$$
\left(\begin{array}{cccc}
\Sigma_{11}(t) & \Sigma_{12}(t) & 0 & 0 \\
0 & \Sigma_{22}(t) & 0 & 0 \\
0 & \Omega_{s}(t) & 0 & 0 \\
0 & 0 & 0 & 0 \\
0 & 0 & 0 & 0 \\
0 & 0 & 0 & 0
\end{array} \|, \quad\right. \text { }
$$

and applying theorem 1 to the block:

$$
\left\|\begin{array}{c}
\Sigma_{22}(t) \\
\Omega_{s}(t)
\end{array}\right\|
$$

we obtain:

$$
\begin{aligned}
& \left(\left\|\begin{array}{cccc}
\Sigma_{11}(t) & \Sigma_{12}(t) & 0 & 0 \\
0 & \hat{\Sigma}_{22}(t) & 0 & 0 \\
0 & 0 & 0 & 0 \\
0 & 0 & 0 & 0 \\
0 & 0 & 0 & 0 \\
0 & 0 & 0 & 0
\end{array}\right\|,\right. \\
& \left.\left\|\begin{array}{cccc}
B_{11}(t) & B_{12}(t) & B_{13}(t) & B_{14}(t) \\
B_{21}(t) & B_{22}(t) & B_{23}(t) & B_{24}(t) \\
B_{31}(t) & B_{32}(t) & B_{33}(t) & B_{34}(t) \\
B_{41}(t) & B_{42}(t) & \Gamma_{a}(t) & 0 \\
0 & \Omega_{s}(t) & 0 & 0 \\
0 & 0 & 0 & 0
\end{array}\right\|\right)
\end{aligned}
$$

In the same way we now apply theorem 1 to the block:

$$
\left\|\begin{array}{cc}
B_{33}(t) & B_{34}(t) \\
\Gamma_{a}(t) & 0
\end{array}\right\|
$$

this lead to:

$$
\left(\left\|\begin{array}{cccc}
\Sigma_{11}(t) & \Sigma_{12}(t) & 0 & 0 \\
0 & \hat{\Sigma}_{22}(t) & 0 & 0 \\
0 & 0 & 0 & 0 \\
0 & 0 & 0 & 0 \\
0 & 0 & 0 & 0 \\
0 & 0 & 0 & 0
\end{array}\right\|\right.
$$

${ }^{1}$ Of course, blocks $B_{i j}$ are changed. 


$$
\left\|\begin{array}{cccc}
B_{11}(t) & B_{12}(t) & B_{13}(t) & B_{14}(t) \\
B_{21}(t) & B_{22}(t) & B_{23}(t) & B_{24}(t) \\
B_{31}(t) & B_{32}(t) & \Gamma_{a_{1}}(t) & 0 \\
B_{41}(t) & B_{42}(t) & 0 & 0 \\
0 & \Omega_{s}(t) & 0 & 0 \\
0 & 0 & 0 & 0
\end{array}\right\|
$$

Finally we apply theorem 1 to the block:

$$
\left\|\begin{array}{cc}
B_{41}(t) & B_{42}(t) \\
\Omega_{s}(t) & 0
\end{array}\right\|
$$

the system is then:

$$
\begin{aligned}
& \left(\begin{array}{cccc}
\Sigma_{11}(t) & \Sigma_{12}(t) & 0 & 0 \\
0 & \Sigma_{22}(t) & 0 & 0 \\
0 & 0 & 0 & 0 \\
0 & 0 & 0 & 0 \\
0 & 0 & 0 & 0
\end{array} \|,\right. \\
& \left.\left\|\begin{array}{cccc}
B_{11}(t) & B_{12}(t) & B_{13}(t) & B_{14}(t) \\
B_{21}(t) & B_{22}(t) & B_{23}(t) & B_{24}(t) \\
B_{31}(t) & B_{32}(t) & \Gamma_{a_{1}}(t) & 0 \\
0 & \Omega_{s_{1}}(t) & 0 & 0 \\
0 & 0 & 0 & 0
\end{array}\right\|\right)
\end{aligned}
$$

This procedure can be repeated. As in Kunkel and Mehrmann's algorithm, this gives rise to a sequence of systems $\left(A_{2}(t), B_{i}(t)\right)$ and to a new sequence of characteristic values $\left(r_{i}, a_{i}, s_{i}\right)$. Let us notice that no rank assumption has been done: our transformations are valid on whole $I$.

Theorem 3 There exists a number $m$ defined by:

$$
m= \begin{cases}\min \left\{i \in \mathbf{N}, s_{i+1}=s_{i}\right\} & \text { if } s_{0} \neq 0 \\ 0 & \text { otherwise }\end{cases}
$$

Proof If $s_{0} \neq 0,\left(s_{i}\right)_{i \in \mathbf{N}}$ is an increasing sequence with $\operatorname{Min}\{n, m\}$ as upper bound.

When $m$ is reached the iterations are stopped.

Proposition 1 When Kunkel and Mehrmann's algorithm can be applied, the number $m$ defined in theorem 3 and the strangeness index defined by Kunkel and Mehrmann are equal.

Proof Applying our generalized algorithm on a system and getting $\Sigma_{11}=I d, \Sigma_{22}=I d, \Gamma_{a_{2}}=I d_{a_{i}}$ and $\Omega_{s_{2}}=I d_{s_{i}}$ for all $i, 0 \leq i \leq m$ is equivalent to say:

$$
\hat{s}_{i+1}-\hat{s}_{\imath}=s_{2} \quad 0 \leq i \leq m
$$

where $\hat{s}$ is our $s$ characteristic value and $s$ the characteristic value defined by Kunkel and Mehrmann.

\section{Full system, index, and singularity}

As seen above our new algorithm can be applied to any system in input. The final form will be called full system. This section is intended to give its properties and to apply it for the solution of the system.

\subsection{Full system}

When $s_{i+1}=s_{\imath}$, we obtain the final equivalent system

$$
\begin{gathered}
\left(\left\|\begin{array}{cccc}
\Sigma_{11}(t) & \Sigma_{12}(t) & 0 & 0 \\
0 & \Sigma_{22}(t) & 0 & 0 \\
0 & 0 & 0 & 0 \\
0 & 0 & 0 & 0 \\
0 & 0 & 0 & 0
\end{array}\right\|,\right. \\
\left.\left\|\begin{array}{cccc}
B_{11}(t) & B_{12}(t) & B_{13}(t) & B_{14}(t) \\
B_{21}(t) & B_{22}(t) & B_{23}(t) & B_{24}(t) \\
B_{31}(t) & B_{32}(t) & \Gamma_{a_{m}}(t) & 0 \\
0 & \Omega_{s_{m}}(t) & 0 & 0 \\
0 & 0 & 0 & 0
\end{array}\right\|\right)
\end{gathered}
$$

Proposition 2 Sequences $\left(a_{i}\right)$ and $\left(s_{i}\right)$ have the following properties:

$$
\begin{aligned}
& a_{i}<a_{i+1} \quad \text { for } i<m, \quad a_{i}=a_{m} \quad \text { for } i \geq m \\
& s_{i+1}>s_{i} \quad \text { for } i<m, \quad s_{i}=s_{m} \quad \text { for } i \geq m
\end{aligned}
$$

In other words, the system (14) completed with the derivative of the third equation:

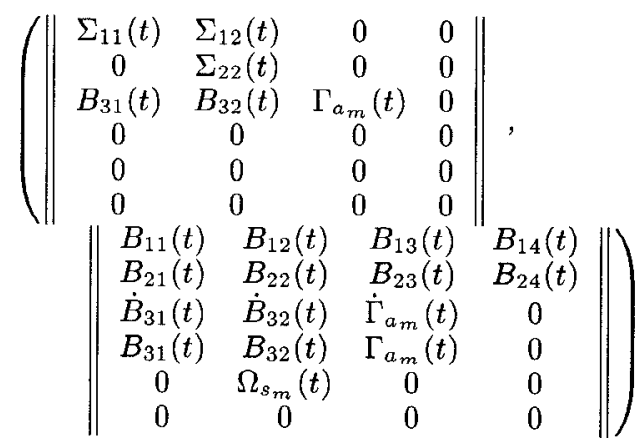

is a system, equivalent to the initial one, where the $O D E$ :

$$
\begin{gathered}
\left(\left\|\begin{array}{cccc}
\Sigma_{11}(t) & \Sigma_{12}(t) & 0 & 0 \\
0 & \Sigma_{22}(t) & 0 & 0 \\
B_{31}(t) & B_{32}(t) & \Gamma_{a_{m}}(t) & 0
\end{array}\right\|\right. \\
\left.\left\|\begin{array}{cccc}
B_{11}(t) & B_{12}(t) & B_{13}(t) & B_{14}(t) \\
B_{21}(t) & B_{22}(t) & B_{23}(t) & B_{24}(t) \\
\dot{B}_{31}(t) & \dot{B}_{32}(t) & \dot{\Gamma}_{a_{m}}(t) & 0
\end{array}\right\|\right)
\end{gathered}
$$

is the "biggest" one, i.e. has the largest possible dimension for an $O D E$ underlying the initial system.

The systems which verify this property are called full systems.

Proof As in the proof of proposition 1, applying our algorithm on a system that fulfills Kunkel and Mehrmann's condition we get $a_{i}=\hat{a}_{i}$ where $\hat{a}$ is our characteristic value and $a$ the characteristic value defined by Kunkel and Mehrmann.

Moreover we know that a system fulfills the condition for the application of Kunkel and Mehrmann's algorithm on whole its definition interval except at some isolated point. Using the "continuity" of $\hat{a}$ and $\hat{s}$, the two equations $a_{i}=\hat{a}_{i}$ and $\hat{s}_{i+1}-\hat{s}_{i}=s_{i}$, and the equations (9) established by Kunkel and Mehrmann, we complete the proof.

Let us look at the proposition in the regular case. To a regular system of dimension $n$, by definition, we can associate an underlying ODE of dimension $n$ [10]. The proposition expresses that the algorithm has computed such an equation. The leads directly to generalize the index as below. 


\subsection{Generalization of the index}

When the initial system is regular, or more generally, has not undetermined component, the ODE (15) involves all the entries of $\dot{x}(t)$. Clearly, in this case, as for the strangeness index of Kunkel and Mehrmann, if $m \neq 0$ or $a_{0} \neq 0$ the differential index is given by $i_{d}=m+1$ (otherwise $i_{d}=0$ ). So the natural extention of definition 1 for the index of any system (1) that we will take is:

$$
i_{d}= \begin{cases}0 & \text { for } m=0, a_{0}=0 \\ m+1 & \text { otherwise }\end{cases}
$$

Another application of form (14) we will see now is that it leads to a natural definition of the singularities of a system (1).

\subsection{Singularities of the system, existence and uniqueness of solutions}

We now look at conditions under which our system is equivalent to an ODE without singularity and an algebraic nonsingular system. For that, we clearly need $\Sigma_{11}(t)$ and $\Gamma_{a_{m}}(t)$ to be regular. Furthermore if $\Omega_{s_{m}}(t)$ is singular even if $\Sigma_{22}(t)$ is regular, the algebraic nonsingular system cannot be obtained using equivalent transformations only. From this we define the singularities of the system as follows.

Definition 3 A system on an interval $I$ has a singularity at point $t_{0} \in I$ if one of the submatrices $\Sigma_{11}\left(t_{0}\right), \Gamma_{a_{m}}\left(t_{0}\right)$ or $\Omega_{s_{m}}\left(t_{0}\right)$ has not a full rank.

Theorem 4 Any system (1) may be put into the equivalent form (14) on the whole of its definition interval. On each sub-interval such that the system has no singularity (14) is equivalent to a system:

$$
\left\{\begin{array}{l}
\dot{x}_{1}(t)+B_{11}(t) x_{1}(t)=g_{1}(t)-B_{13}(t) x_{3}(t) \\
x_{2}(t)=g_{2}(t) \\
0=g_{3}(t)
\end{array}\right.
$$

where the inhomogeneity is determined by $f(t), \dot{f}(t), \ldots$, $f^{(m)}(t)$.

Lemma 1 Let $(A(t), B(t))$ and $(\tilde{A}(t), \bar{B}(t))$ satisfy the algebraic relation (5), sequences $\left(r_{i}, a_{i}, s_{i}\right)$ generated by $(A(t), B(t))$ and $\left(\tilde{r}_{i}, \tilde{a}_{i}, \tilde{s}_{i}\right)$ generated by $(\tilde{A}(t), \tilde{B}(t))$ are the same.

Proof Our characteristic values are defined on the whole of the definition interval but are equal to the Kunkel and Merhmann ones when the latter are defined. Thus, by $[13$, theorem 7 , p5], we have:

$$
r_{0}=\tilde{r}_{0}, \quad a_{0}=\tilde{a}_{0}, \quad s_{0}=\tilde{s}_{0}
$$

Assume now that $(A(t), B(t))$ and $(\tilde{A}(t), \tilde{B}(t))$ are in the form (11) which we rewrite:

$$
\begin{aligned}
& \left(\left\|\begin{array}{ccccc}
\Sigma_{11}(t) & \Sigma_{12}(t) & 0 & 0 & 0 \\
0 & \Sigma_{22}(t) & 0 & 0 & 0 \\
0 & 0 & 0 & 0 & 0 \\
0 & 0 & 0 & 0 & 0 \\
0 & 0 & 0 & 0 & 0
\end{array}\right\|,\right. \\
& \left.\left\|\begin{array}{ccccc}
B_{11}(t) & B_{12}(t) & B_{13}(t) & B_{14}(t) & B_{15}(t) \\
B_{21}(t) & B_{22}(t) & B_{23}(t) & B_{24}(t) & B_{25}(t) \\
B_{31}(t) & B_{32}(t) & \Gamma_{a}(t) & 0 & 0 \\
0 & \Omega_{s}(t) & 0 & 0 & 0 \\
0 & 0 & 0 & 0 & 0
\end{array}\right\|\right)
\end{aligned}
$$

By assumption, there exist analytic and nonsingular $P(t)$, $Q(t)$ such that (omitting arguments):

$$
P \tilde{A}=A Q \text { and } P \tilde{B}=B Q+A \dot{Q}
$$

Using a similar method to the one used in [13, proof of theorem 10, p12], we consider the first previous identity, after some transformations we have:

$$
\begin{aligned}
& \left\|\begin{array}{ccccc}
* & * & 0 & 0 & 0 \\
* & * & 0 & 0 & 0 \\
P_{31} \dot{\Sigma}_{11} & P_{31} \tilde{\Sigma}_{12}+P_{32} \tilde{\Sigma}_{22} & 0 & 0 & 0 \\
P_{41} \dot{\Sigma}_{11} & P_{41} \bar{\Sigma}_{12}+P_{42} \tilde{\Sigma}_{22} & 0 & 0 & 0 \\
P_{51} \dot{\Sigma}_{11} & P_{51} \Sigma_{12}+P_{52} \Sigma_{22} & 0 & 0 & 0
\end{array}\right\|= \\
& \| \begin{array}{ccccc}
* & * & \Sigma_{11} Q_{13}+\Sigma_{12} Q_{23} & \Sigma_{11} Q_{14}+\Sigma_{12} Q_{24} & \Sigma_{11} Q_{15}+\Sigma_{12} Q_{25} \\
* & * & \Sigma_{22} Q_{23} & \Sigma_{22} Q_{24} & \Sigma_{22} Q_{25} \\
0 & 0 & 0 & 0 & 0 \\
0 & 0 & 0 & 0 & 0 \\
0 & 0 & 0 & 0 & 0
\end{array}
\end{aligned}
$$

Thus:

$$
\begin{gathered}
P_{31}=P_{41}=P_{51}=P_{32}=P_{42}=P_{52}=0 \text { and } \\
Q_{23}=Q_{24}=Q_{25}=Q_{13}=Q_{14}=Q_{15}=0
\end{gathered}
$$

Writting the last three row blocks of the second identity, we find:

$$
\begin{gathered}
P_{53}=P_{54}=P_{43}=0, \\
Q_{34}=Q_{35}=Q_{21}=0 \text { and } \\
P_{44} \tilde{\Omega}_{s}=\Omega_{s} Q_{22}
\end{gathered}
$$

Replacing these zero blocks in $P$ and $Q$ we see that $P_{44}$ and $Q_{22}$ must be nonsingular, thus $\left(0, \tilde{\Omega}_{s}\right)$ and $\left(0, \Omega_{s}\right)$ are algebraically equivalent.

We define now $\hat{P}$ by:

$$
\hat{P}=\left\|\begin{array}{cccccc}
P_{11} & P_{12} & P_{13} & P_{14} & 0 & P_{15} \\
P_{21} & P_{22} & P_{23} & P_{24} & 0 & P_{25} \\
0 & 0 & P_{33} & P_{34} & 0 & P_{35} \\
0 & 0 & 0 & P_{44} & 0 & P_{45} \\
0 & 0 & 0 & \dot{P}_{44} & P_{44} & 0 \\
0 & 0 & 0 & 0 & 0 & P_{55}
\end{array}\right\| .
$$

We have

$$
\hat{P} \tilde{A_{1}}=A_{1} Q \text { and } \hat{P} \tilde{B}_{1}=B_{1} Q+A_{1} \dot{Q}
$$

where $\left(A_{1}, B_{1}\right)$ and $\left(\tilde{A}_{1}, \tilde{B}_{1}\right)$ are in the form:

$$
\begin{gathered}
\left(\left\|\begin{array}{ccccc}
\Sigma_{11}(t) & \Sigma_{12}(t) & 0 & 0 & 0 \\
0 & \Sigma_{22}(t) & 0 & 0 & 0 \\
0 & 0 & 0 & 0 & 0 \\
0 & 0 & 0 & 0 & 0 \\
0 & \Omega_{s}(t) & 0 & 0 & 0 \\
0 & 0 & 0 & 0 & 0
\end{array}\right\|,\right. \\
\\
\left.\left\|\begin{array}{ccccc}
B_{11}(t) & B_{12}(t) & B_{13}(t) & B_{14}(t) & B_{15}(t) \\
B_{21}(t) & B_{22}(t) & B_{23}(t) & B_{24}(t) & B_{25}(t) \\
B_{31}(t) & B_{32}(t) & \Gamma_{a}(t) & 0 & 0 \\
0 & \Omega_{s}(t) & 0 & 0 & 0 \\
0 & \dot{\Omega}_{s}(t) & 0 & 0 & 0 \\
0 & 0 & 0 & 0 & 0
\end{array}\right\|\right)
\end{gathered}
$$

which is similar to the form (12). Thus, if we put two algebraically equivalent systems into the form (13), we have shown that the new systems are algebraically equivalent. This completes the proof. 
Proof (of theorem 4): On every interval such that the system has no singularity, (14) is equivalent to a system:

$$
\begin{gathered}
\left(\left\|\begin{array}{cccc}
I d & \Sigma_{12}(t) & 0 & 0 \\
0 & \Sigma_{22}(t) & 0 & 0 \\
0 & 0 & 0 & 0 \\
0 & 0 & 0 & 0 \\
0 & 0 & 0 & 0
\end{array}\right\|,\right. \\
\left.\left\|\begin{array}{cccc}
B_{11}(t) & B_{12}(t) & B_{13}(t) & B_{14}(t) \\
B_{21}(t) & B_{22}(t) & B_{23}(t) & B_{24}(t) \\
B_{31}(t) & B_{32}(t) & I d_{a_{m}} & 0 \\
0 & I d_{s_{m}} & 0 & 0 \\
0 & 0 & 0 & 0
\end{array}\right\|\right)
\end{gathered}
$$

Using transformations (5) we put this system into the equivalent form:

$$
\begin{gathered}
\left(\left\|\begin{array}{cccc}
I d & 0 & 0 & 0 \\
0 & \Sigma_{22}(t) & 0 & 0 \\
0 & 0 & 0 & 0 \\
0 & 0 & 0 & 0 \\
0 & 0 & 0 & 0
\end{array}\right\|,\right. \\
\left.\left\|\begin{array}{cccc}
B_{11}(t) & 0 & 0 & B_{14}(t) \\
B_{21}(t) & 0 & 0 & B_{24}(t) \\
0 & 0 & I d_{a_{m}} & 0 \\
0 & I d_{s_{m}} & 0 & 0 \\
0 & 0 & 0 & 0
\end{array}\right\|\right)
\end{gathered}
$$

Lemma 1 ensures sequences $\left(r_{i}, a_{i}, s_{i}\right)$ of this last system to be identical to the ones of the initial system. Thus, it follows from proposition 2 that:

$$
\text { for } i \geq m, a_{i}=a_{m} \text { and } s_{i}=s_{m}
$$

Applying our algorithm we see that this is possible only if:

$$
\operatorname{Rank}\left(B_{24}(t)\right)=\operatorname{Rank}\left(B_{21}(t)\right)=0
$$

Applying (6) where $E q_{i}$ and $E q_{j}$ are respectively the second and fourth block of equations of the last system and $a(t)$ is equal to $-\Sigma_{22}(t)$, we can rewrite the system:

$$
\left(\left\|\begin{array}{ccc}
I d & 0 & 0 \\
0 & 0 & 0 \\
0 & 0 & 0
\end{array}\right\|,\left\|\begin{array}{ccc}
B_{11}(t) & 0 & B_{14}(t) \\
0 & I d_{a_{m}+s_{m}} & 0 \\
0 & 0 & 0
\end{array}\right\|\right)
$$

which is into the form (17).

Once the form (17) is obtained we can immediately conclude concerning the existence and uniqueness of the solutions of the system.

Corollary 1 On all interval $\tilde{I}$ such that the system has no singularity, the system has at least one solution if and only if $f(t)$ is $C^{m}(\mathbf{R})$ and satisfies $g_{3}(t)=0$.

An initial condition at $t_{0} \in \tilde{I}$ is consistent if and only if it satisfies $x_{2}\left(t_{0}\right)=g_{2}\left(t_{0}\right)$.

$A$ solution is uniquely determined by its value at $t_{0} \in \tilde{I}$ if and only if $\operatorname{Dim}\left(x_{1}(t)\right)+\operatorname{Dim}\left(x_{2}(t)\right)=p$.

\section{The polynomial case}

In practice, it is not easy to implement theorems 1 and 2 for analytic functions. On the contrary, the polynomial case

$$
A(t) \dot{x}(t)+B(t) x(t)=f(t)
$$

where $A(t)$ and $B(t)$ have polynomial coefficients, may be implemented. We give in this section the polynomial versions of theorems 1 and 2 and we show how a new set of singularities, smallest than the one given in section 4.3 , can be computed.

We assume in this section that the coefficients of $A(t)$ and $B(t)$ are in $\mathbf{K}[t]$ where $\mathbf{K}[t]$ is the polynomial ring on $\mathbf{K}$, subfield of $\mathbf{R}$.

A nonsingular matrix $M(t) \in \mathbf{K}^{(n, n)}[t]$ is in Hermite normal form if it is upper triangular and if its entries satisfy:

$$
\begin{aligned}
& m_{i i}(t) \text { is monic for } i \in[1 . . n] \\
& \operatorname{Deg}\left(m_{j i}\right)(t)<\operatorname{Deg}\left(m_{i i}(t)\right) \text { for } i \in[1 . . n], j \in[1 . . i-1]
\end{aligned}
$$

Theorem 5 Given a nonsingular matrix $M(t)$, components of which are in $\mathbf{K}[t]$, there exists a nonsingular square matrix $P(t)$ such that $P(t) M(t)$ is in Hermite normal form.

Theorem 6 Given a r-ranked matrix $M(t)$ components of which are in $\mathbf{K}[t]$, there exist two matrices $P(t)$ and $Q(t)$, such that:

$$
\begin{aligned}
& P(t) M(t) Q(t)=\left\|\begin{array}{cc}
H(t) & 0 \\
0 & 0
\end{array}\right\| \text { and } \\
& \operatorname{Det}(P(t)) \in \mathbf{K}^{*}, \operatorname{Det}(Q(t)) \in \mathbf{K}^{*}
\end{aligned}
$$

where $H(t)$ is a Hermite square matrix of rank and order $r$.

The proof of theorem 5 can be found in [6, (II, chap. 6)] and the proof of theorem 6 can be done by two applications of theorem 5 on the matrix then on its transpose.

Moreover there exist polynomial time algorithms that compute the Hermite normal form.

Given these two matrix transfomations, our agorithm described in section 3 applies directly to the simpler case of polynomial coefficients, but it gives rise to a new reduction on the coefficient degrees. Let us see on an example.

\section{Example}

$$
\left\{\begin{array}{l}
t x(t)=f_{1}(t) \\
t^{2} x(t)=f_{2}(t)
\end{array}\right.
$$

The algorithm computes the equivalent system:

$$
\left\{\begin{array}{l}
t x(t)=f_{1}(t) \\
t x(t)=\frac{1}{2}\left(-t f_{1}(t)+\dot{f}_{2}(t)\right) \\
0=t^{2} f_{1}(t)-t \dot{f}_{2}(t)+2 f_{2}(t)
\end{array}\right.
$$

and stops because $\Omega_{0}(t)=\left\|t^{2}\right\|$ and $\Omega_{1}(t)=\|t\|$ have the same order.

Nevertheless, $\operatorname{Deg}\left(\operatorname{Det}\left(\Omega_{1}(t)\right)\right)<\operatorname{Deg}\left(\operatorname{Det}\left(\Omega_{0}(t)\right)\right)$, and if we take a step forward in the algorithm we find:

$$
\left\{\begin{array}{l}
t x(t)+x=\frac{1}{2}\left(-f_{1}(t)-t \dot{f}_{1}(t)+f_{2}^{(2)}(t)\right) \\
x(t)=\frac{1}{2}\left(-3 f_{1}(t)-t \dot{f}_{1}(t)+f_{2}^{(2)}(t)\right) \\
0=t f_{1}(t)+\frac{1}{2}\left(t^{2} \dot{f}_{1}(t)-t f_{2}^{(2)}(t)+\dot{f}_{2}(t)\right) \\
0=t^{2} f_{1}(t)-t \dot{f}_{2}(t)+2 f_{2}(t)
\end{array}\right.
$$

The second equation of system (19) gives a solution on the whole of $\mathbf{R}$ whereas definition 3 gives 0 as a singularity.

This reduction on the coefficients degrees exists in the polynomial case because the derivative of a polynomial of 
degree $d$ is a polynomial of degree $d-1$. If we take this reduction into consideration, we can apply our algorithm and compute the sequences $\left(A_{2}(t), B_{i}(t)\right)$ up to $\tilde{m} \geq m$ defined by:

$$
\begin{aligned}
\tilde{m}=\operatorname{Min}\{\quad & i \in \mathbf{N}, s_{i+1}=s_{i} \text { and } \\
& \left.\operatorname{Deg}\left(\operatorname{Det}\left(\Omega_{s_{i+1}}\right)\right)=\operatorname{Deg}\left(\operatorname{Det}\left(\Omega_{s_{i}}\right)\right)\right\}
\end{aligned}
$$

This reduction allows us to find a smaller set of singularities. Nevertheless, it may be possible that we find a more restrictive set of conditions on $f(t)$. Thus, applying the algorithm up to $\tilde{m}$, we have just found a characterization of $f(t)$ which gives a smaller set of singularities.

\section{Implementation}

We have implemented our algorithm in the polynomial case with the system MAPLE. Here are examples of executions. The function RedSyst computes the full system, at each step $i$ of the algorithm the program prints the current system $\left(A_{i}(t), B_{i}(t), f_{i}(t)\right)$.

\section{Example 1}

$$
\left\{\begin{array}{l}
t \dot{x}_{2}(t)+x_{1}(t)=f_{1}(t) \\
x_{2}(t)=f_{2}(t)
\end{array}\right.
$$

$>$ RedSyst(A,B,t);

$$
\begin{gathered}
{\left[\begin{array}{ll}
t & 0 \\
0 & 0
\end{array}\right],\left[\begin{array}{ll}
0 & 1 \\
1 & 0
\end{array}\right],\left[\begin{array}{l}
\mathrm{f} 1(t) \\
\mathrm{f} 2(t)
\end{array}\right]} \\
\cdots \cdots \cdots \\
{\left[\left[\begin{array}{ll}
t & 0 \\
0 & 0
\end{array}\right],\left[\begin{array}{ll}
0 & 1 \\
1 & 0
\end{array}\right],\left[\begin{array}{l}
\mathrm{fl}(t) \\
\mathrm{f} 2(t)
\end{array}\right]\right.}
\end{gathered}
$$

Example 2 (see figure 1)

$$
\left\{\begin{array}{l}
t \dot{x}(t)=f_{1}(t) \\
t^{2} x(t)=f_{2}(t)
\end{array}\right.
$$

In this latter case a solution is explicitely computed that is given by the second row of the output matrices; the eventual problem at $t=0$ arises for particular $f(t)$ that do not satisfy to the equations given by the last two rows.

\section{Conclusion}

Our new algorithm allows the reduction of any system (1) on the whole interval of definition. Once the system is reduced, we can conclude to the existence and uniqueness of solutions provided some assumptions are satisfied. Our assumptions are less restrictive than Kunkel and Mehrmann's ones and induce us to define a set of singularities for a system (1). Moreover, in the case of polynomial coefficients, the algorithm can be implemented and gives a smaller set of singularities.

Nevertheless, our algorithm can be improved considering the algebraic conditions on $f(t)$ and its derivatives. Let's look at the example:

$$
\left\{\begin{array}{l}
t x(t)=f_{1}(t) \\
t^{2} x(t)=f_{2}(t)
\end{array}\right.
$$

\section{$>\operatorname{RedSyst}(A, B, t)$;}

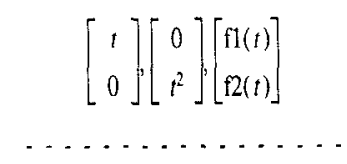

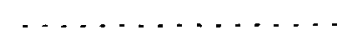

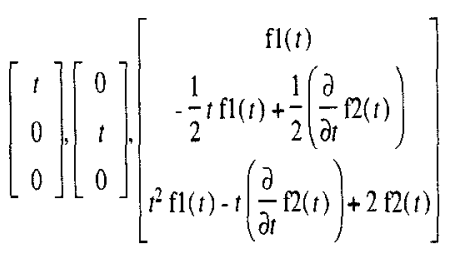

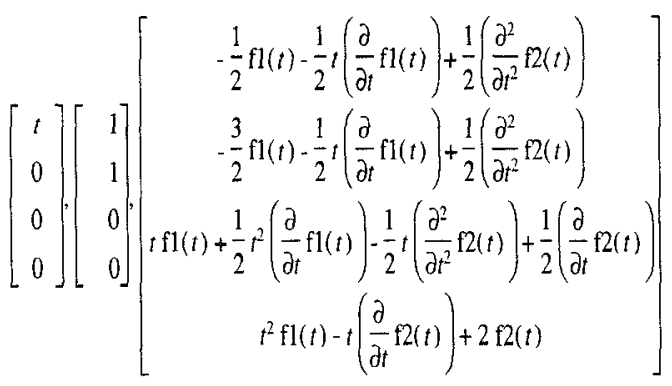

$$
\left[\left[\begin{array}{l}
t \\
0 \\
0 \\
0
\end{array}\right],\left[\begin{array}{c}
1 \\
1 \\
0 \\
0
\end{array}\right] \cdot\left[\begin{array}{c}
-\frac{1}{2} \mathrm{f} 1(t)-\frac{1}{2} t\left(\frac{\partial}{\partial t} \mathrm{f} 1(t)\right)+\frac{1}{2}\left(\frac{\partial^{2}}{\partial t^{2}} \mathrm{f}(t)\right) \\
-\frac{3}{2} \mathrm{fl}(t)-\frac{1}{2} t\left(\frac{\partial}{\partial t} \mathrm{fl}(t)\right)+\frac{1}{2}\left(\frac{\partial^{2}}{\partial t^{2}} \mathrm{f} 2(t)\right) \\
f \mathrm{f} 1(t)+\frac{1}{2} t^{2}\left(\frac{\partial}{\partial t} \mathrm{fl}(t)\right)-\frac{1}{2} t\left(\frac{\partial^{2}}{\partial t^{2}} \mathrm{f} 2(t)\right)+\frac{1}{2}\left(\frac{\partial}{\partial t} \mathrm{f} 2(t)\right) \\
t^{2} \mathrm{fl}(t)-t\left(\frac{\partial}{\partial t} \mathrm{f}(t)\right)+2 \mathrm{f} 2(t)
\end{array}\right]\right]
$$

Figure 1: Execution (example 2)

The algorithm (in the polynomial case) gives the equivalent system:

$$
\left\{\begin{array}{l}
\dot{x}(t)=-2 \dot{f}_{1}(t)-\frac{t}{2} f_{1}^{(2)}(t)+\frac{1}{2} f_{2}^{(3)}(t) \\
x(t)=-\frac{1}{2}\left(3 f_{1}(t)+t \dot{f}_{1}(t)-f_{2}^{(2)}(t)\right) \\
0=-f_{1}(t)-2 t \dot{f}_{1}(t)-\frac{t^{2}}{2} f_{1}^{(2)}(t)+\frac{t}{2} f_{2}^{(3)}(t) \\
0=t f_{1}(t)+\frac{1}{2}\left(t^{2} \dot{f}_{1}(t)-t f_{2}^{(2)}(t)+\dot{f}_{2}(t)\right) \\
0=t^{2} f_{1}(t)-t \dot{f}_{2}(t)+2 f_{2}(t)
\end{array}\right.
$$

where the second equation gives the solution, the first equation is the derivative of the second one, and the three last equations are algebraic conditions on $f(t)$ and its derivatives. These last equations are not independent. In fact we only need the last equation:

$$
0=t^{2} f_{1}(t)-t \dot{f}_{2}(t)+2 f_{2}(t)
$$

to describe the algebraic conditions on $f(t)$ and its derivatives. 


\section{References}

[1] S.L. CAMPBELl, A general form for solvable linear time varying singular systems of differential equations, SIAM J. Math. Anal., 18 (1987), 1101-1115.

[2] S.L. CAMPBELL, The numerical solution of higher index linear time varying singular systems of differential equations, SIAM J. Sci. Stat. Comput., 6 (1988), 334-348.

[3] S.L. CAMPBELL and L.R.PETzOLD, Canonical forms and solvable singular systems of differential equations, SIAM J. Alg. Disc. Meth., 4 (1983), 517-521.

[4] Fliess, LeVIne and Rouchon, Index of an implicit timevarying linear differential equation, Proc. of the 1rst european control conference, Hermes 1991.

[5] Fliess, Levine and Rouchon, Index of a general differential algebraic implicit system, Proc. of MTNS-91, Kobe, Japan, 289-294, Mita press, 1992.

[6] F.R. Gantmachen, Théorie des matrices, Dunod, Paris France, 1966.

[7] C.W. Gear and L.R.Petzold, Differential algebraic systems and matrix pencils, Proc. Conference on Matrix Pencils, Pitea Sweden (1982), Lecture Notes in Mathematics 973.

[8] C.W. Gear and L.R.PeTzold, ODE methods for the solution of differential algebraic systems, SIAM J. Numer. Anal., 21 (1984), 716-728.

[9] C.W. GEAR, DAE: ODE with constraints and invariants, Workshop on Numerical Methods for Ordinary Differential Equations, L'Aquila Italy, Sept. 15-18, 1987.

[10] C.W. GEAR, Differential-algebraic equation index transformations, SIAM J. Sci. Stat. Comput., 9 (1988), 39-47.

[11] C.W. GeAR, Differential algebraic equations, indices, and Integral algebraic equations, SIAM J. Numer. Anal., 27 (1990), 1527-1534.

[12] R. KANNAN, Solving systems of linear equations over polynomials, Theoretical Computer Science, 39 (1985), 69-88.

[13] P. Kunkel and V Mehrmann, Canonical Forms for Linear Differantial-Algebraic Equations with Variable Cofficients, TR 69, Institut fr Geometrie und Praktische Mathematik, RWTH Aachen Germany, to appear in J. Comp. Appl. Math.

[14] P. Kunkel and V Mehrmann, A New Look at Matrix Pencils, Linear Algebra and its Applications, 24 (1992).

[15] M.P. QuŔE, Etude de la théorie des faisceaux de matrices et application la simplification d'équations différentielles ordinaires du type : $A \dot{x}(t)+B x(t)=f(t)$, Rapport de DEA de Mathématiques Appliquées, INPG-UJF Grenoble France, Juin 1993.

[16] P.J. Rabier and W.C. RheinBoldt, A geometric treatement of implicit differential-algebraic equations, J. of Diff. Eq., 109, 110-146 (1994).

[17] P.J. RABIER and W.C. RHEINBOLDT, On impasse points of quasilinear differential algebraic equations, Tech. Report ICMA-92-171, 1992.

[18] P.J. RABIER and W.C. RHEINBOLDT, On the computation of impasse points of quasilinear differential algebraic equations, Preprint, 1992.

[19] P.J. RABIER and W.C. RheinboldT, Classical and generalized solutions of time-dependent linear differential algebraic equations, Tech. Report ICMA-93-193, 1993.

[20] P.J. R.ABIER and W.C. RHEINBOLDT, Time-dependent linear DAEs with discontinuous imputs, Tech. Report ICMA-94$186,1994$.

[21] L.M. Silverman and R.S.BuCY, Generalizations of a theorem of Dolezal, Mathematical Systems Theory, 4 (1970), 334-339.
[22] A. SzatKowsKI, Geometric characterization of singular differential algebraic equations, Int. J. Syst. Sci., Vol. 23, No. 2, 167-186 (1992). 